\title{
Electrostatic Potentials of Nanostructures Revealed in 3D by Electron Holographic Tomography
}

\author{
Daniel Wolf
}

Triebenberg Laboratory, Institute of Structure Physics, Technische Universität Dresden, D-01062 Dresden, Germany

Electron holographic tomography (EHT), i.e., off-axis electron holography $(\mathrm{EH})$ combined with electron tomography (ET) in the transmission electron microscope (TEM), allows quantitative 3D mapping of electrostatic potentials with a lateral resolution of a few (1-10) nanometers [1,2]. The thereby reconstructed potentials of the nanostructures reveal their morphology and their intrinsic structure including the chemical composition; in specific cases, their functional potentials, such as the built-in voltage across $\mathrm{p}-\mathrm{n}$ junctions important for the functionality of semiconductors devices (e.g. transistors), can also be analyzed [3]. In particular the latter possibility is unique and beyond the capabilities of scanning (S)TEM tomography.

Fig. 1 illustrates the three main steps of EHT: Acquisition of a hologram tilt series, holographic reconstruction of the phase image tilt series, and tomographic reconstruction of the 3D potential. The phase images tilt series represents a very suitable dataset for tomographic reconstruction, because a phase image is equal to the projected object potential times a well-known interaction constant.

In our laboratory, we have widely automated the EHT workflow [3], which is much more laborious as for conventional ET. This enables revealing 3D potentials within a relatively small amount of time (1-2 days). For tomographic reconstruction, we use a self-developed algorithm, the Weighted Simultaneous Iterative Reconstruction Technique (W-SIRT). W-SIRT tomograms provide higher resolution and fidelity than those obtained by conventional reconstruction techniques of the same dataset [4].

It is a decisive advantage over $2 \mathrm{D}$ potential mapping with $\mathrm{EH}$ that one can measure with EHT the potential inside the sample, underneath the dead layer and not corrupted by thickness variations. This is demonstrated in Fig. 2, which shows the 3D potential distribution in an n-type MOSFET (AMD 90nm technology). The rod-shaped specimen was prepared by focused ion beam (FIB). On the one hand, as shown in Fig. 2 (b), the different materials within the device are visible due to their different mean inner potentials (MIP). On the other hand, the highly n-doped regions can be clearly identified in the p-type substrate due to the built-in voltage $V_{p n}$ across the p-n junction (Fig. 2 (c)). The line profiles (Fig. 2 $(\mathrm{d}, \mathrm{e}))$ provide values for the MIP of $\mathrm{Si}(12.2 \mathrm{~V})$ and $\mathrm{SiO}_{2}(10.1 \mathrm{~V})$, as well as for $\mathrm{V}_{\mathrm{pn}}(0.8 \mathrm{~V})$ measured inside the rod. These values are in excellent agreement with those in literature supporting the quantitative character of EHT.

References:

[1] PA Midgley and RE Dunin-Borkowski, Nature Materials 8, (2009), p. 271.

[2] D Wolf, H Lichte, G Pozzi, P Prete and N Lovergine, Applied Physics Letters 98 (2011), p. 264103.

[3] D Wolf, A Lubk, H Lichte and H Friedrich, Ultramicroscopy 110 (2010), p. 390.

[4] D Wolf, A Lubk and H Lichte, Ultramicroscopy submitted (2013).

[6] The research leading to these results has received funding from the European Union Seventh Framework Programme, Grant Agreement 312483 - ESTEEM2 (Integrated Infrastructure Initiative - I3). 
Electron hologram tilt series

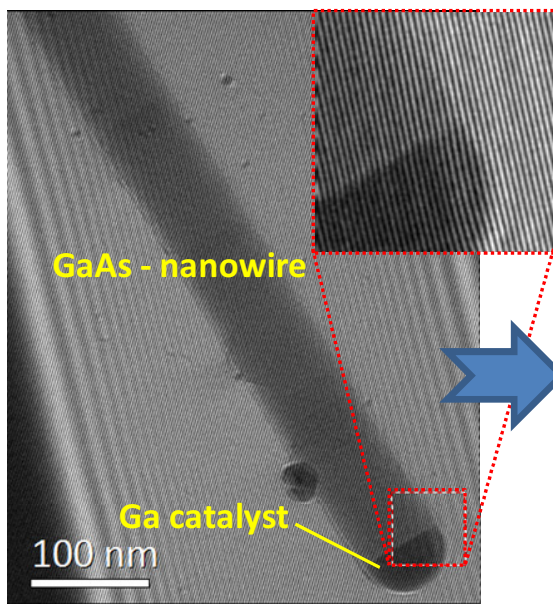

Phase image tilt series

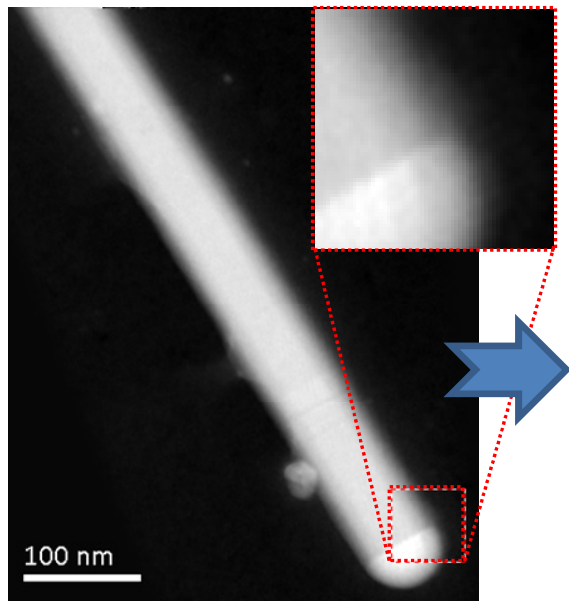

3D electrostatic potential
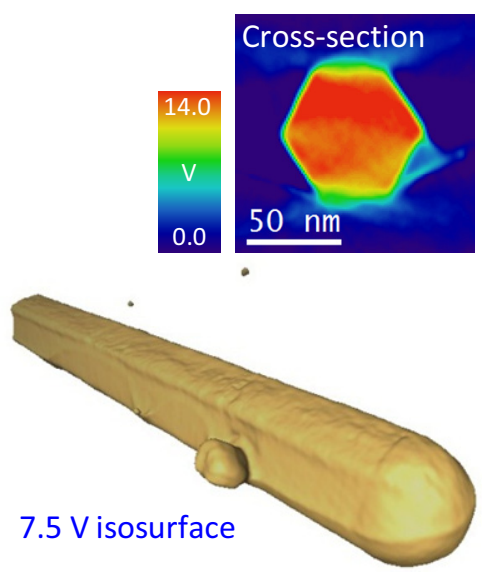

Figure 1. Procedure of electron holographic tomography. A hologram tilt series is acquired in the TEM. Then, the tilt series of the entire object exit waves (amplitude and phase) is reconstructed from these holograms in the computer. Finally, the phase tilt series is used for the tomographic 3D reconstruction of electrostatic potential. (Sample courtesy of G. Koblmueller, TU Munich)

(a)

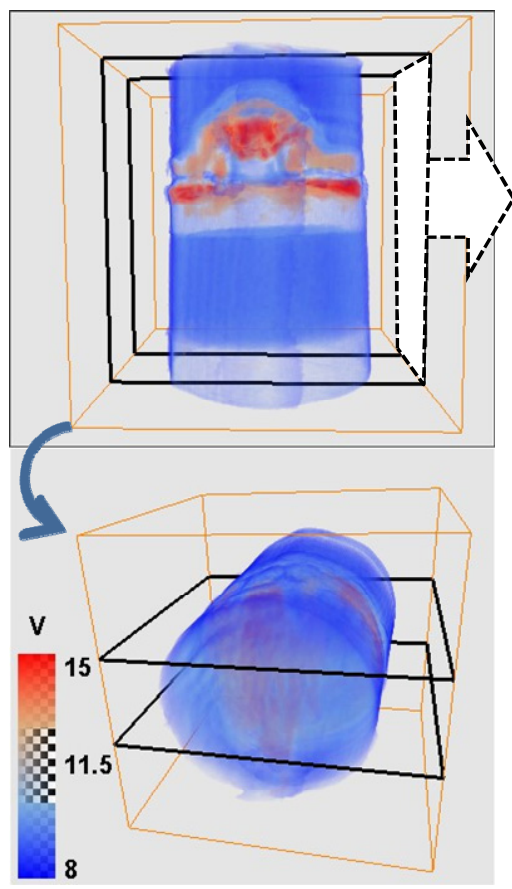

(b)
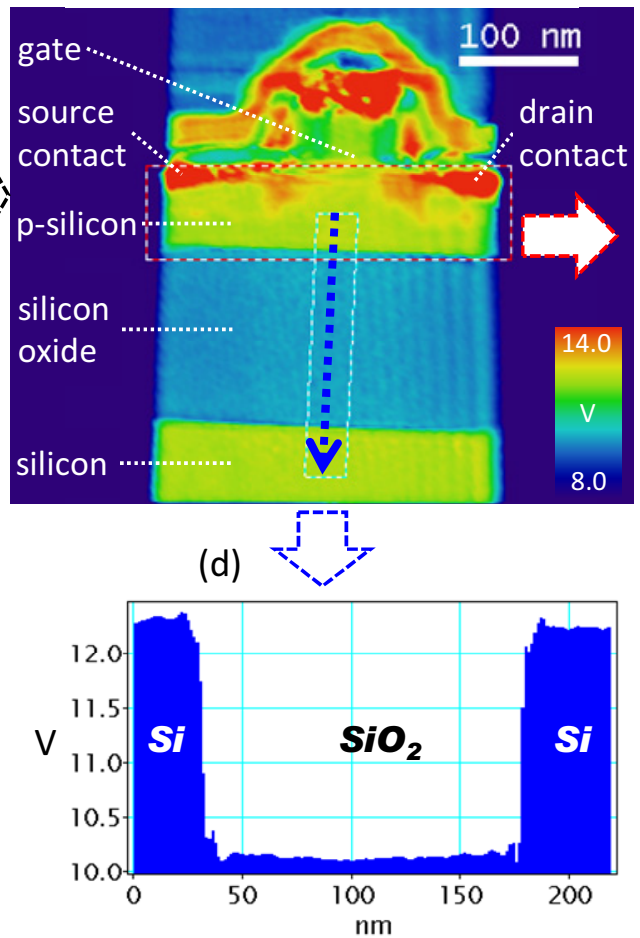

(c)
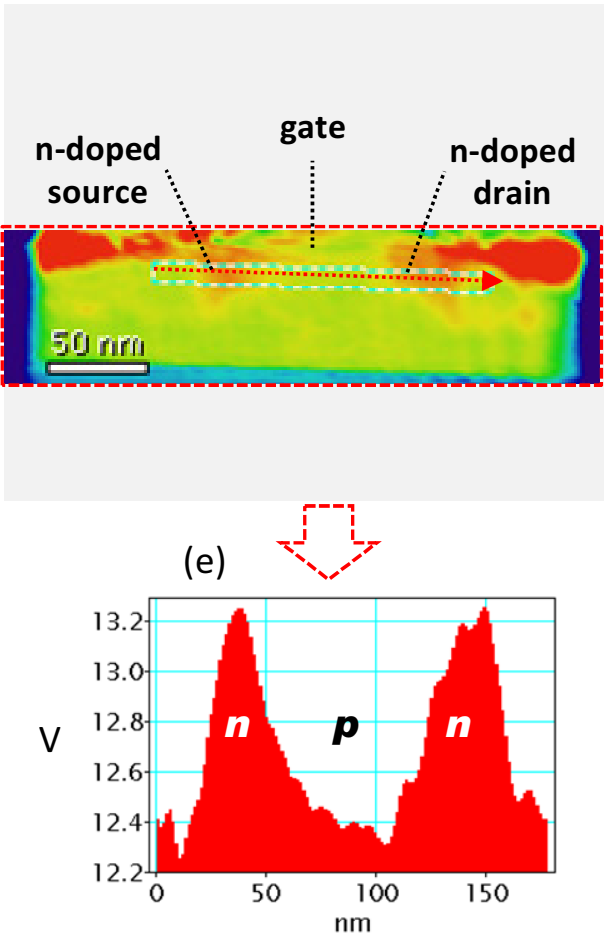

Figure 2. 3D reconstruction of a FIB-prepared sample of an n-type MOSFET (AMD 90nm technology). (a) Volume texture rendering the 3D potential distribution. (b) 2D potential map obtained by averaging over the region between the two planes indicated in (a). (c) p-type substrate with the highly n-doped regions (red). (d,e) Line profiles showing the mean inner potentials of $\mathrm{Si}(12.2 \mathrm{~V})$ and $\mathrm{SiO}_{2}$ $(10.1 \mathrm{~V})$, as well as the built-in voltage $\mathrm{V}_{\mathrm{pn}}$ between $\mathrm{p}$ - and n-type silicon $(0.8 \mathrm{~V})$. (Sample courtesy of H. J. Engelmann, GlobalFoundries, Dresden) 